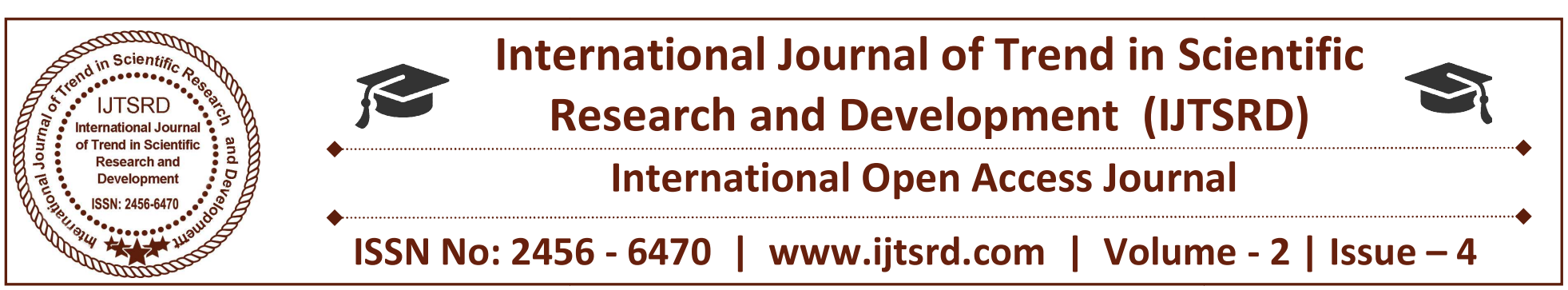

\title{
A Situation Aware Navigation System
}

\author{
Ajinkya Gurav ${ }^{1}$, Sneha Botalji ${ }^{1}$, Rohan Lagare ${ }^{1}$, Rashmi Vaidya ${ }^{1}$, Vijayalaxmi Kanade ${ }^{2}$ \\ ${ }^{1}$ Student, ${ }^{2}$ Professor \\ Computer Engineering, Pune Vidhyarthi Griha's College of Engineering and Technology, \\ Pune, Maharashtra, India
}

\begin{abstract}
When emergencies happen, navigation services that guide people to exits while keeping them away from emergencies are critical in saving lives. To achieve timely emergency navigation, early and automatic detection of potential dangers, and quick response with safe paths to exits are the core requirements, both of which rely on continuous environment monitoring and reliable data transmission. Wireless / sensor networks (WSNs) are a natural choice of the infrastructure to support emergency navigation services, given their relatively easy deployment and affordable costs, and the ability of ubiquitous sensing and communication. Although many efforts have been made to WSN-assisted emergency navigation, almost all existing works neglect to consider the hazard levels of emergencies and the evacuation capabilities of exits. Without considering such aspects, existing navigation approaches may fail to keep people farther away from emergencies of high hazard levels and would probably encounter congestions at exits with lower evacuation capabilities. In this proect, we propose a situation-aware emergency navigation system, which takes the hazard levels of emergencies and the evacuation capabilities of exits into account and provides the mobile users the safest navigation paths accordingly By guiding users following the descend gradient of the hazard potential field, System thereby can thereby achieve guaranteed success of navigation and provide optimal safety.
\end{abstract}

\section{INTRODUCTION}

As emergency or dangerous situations change, it becomes necessary to frequently update the route

plans for the guided users. Path dynamics in traditional packet routing process typically occurs between delivering different packets, while such dynamics in the navigation process might exist all along for guiding single individuals. People may even move backward to seek broader opportunities under varied situations.

There have been attempts made at guiding navigation using WSNs. Most, if not all, existing approaches assume the availability of locations on each sensor node. Knowing the locations of dangerous areas, the sensor network can perform easy and efficient route calculations to navigate internal users out of the emergency area. The location information, however, may not always be available in many realistic situations where emergency guidance are needed, for example, in an underground tunnel or coal mine, a complex indoor area, and so on. The requirement of location information largely constrains the applicability of existing approaches to location-free environments. In addition, existing approaches usually do not specifically consider the impact of variations of dangerous areas, for example, the expansion, shrinking, or disappearing of areas which is deemed dangerous. In reality, such variations often degrade the effectiveness of existing designs or even overwhelm them. So far they haven't take evacuation capability of exits into account which makes big impact in real time.

\section{Overview:}

Recent advances in wireless sensor network (WSN) technologies provide us with the ability to offer 
pervasive usage of sensor networks widely deployed over the space of interest. The increasing study of WSNs aims at enabling computers to better serve people by automatically monitoring and interacting with the physical world. Existing works, however, largely focus on developing sensor network systems principally providing remote data collection. The possibility of interactions between the users and their physical environment is overlooked.

Such interactions could significantly expand the capability of WSNs and thereby enhance their usability.

When emergencies happen, navigation services that guide people to exits while keeping them away from emergencies are critical in saving lives. To achieve timely emergency navigation, early and automatic detection of potential dangers, and quick response with safe paths to exits are the core requirements, both of which rely on continuous environment monitoring and reliable data transmission. Wireless sensor networks (WSNs) are a natural choice of the infrastructure to support emergency navigation services, given their relatively easy deployment and affordable costs, and the ability of ubiquitous sensing and communication.

Although many efforts have been made to WSNassisted emergency navigation, almost all existing works neglect to consider the hazard levels of emergencies and the evacuation capabilities of exits. Without considering such aspects, existing navigation approaches may fail to keep people farther away from emergencies of high hazard levels and would probably encounter congestions at exits with lower evacuation capabilities.

\section{Brief Description:}

We consider the scenario of navigating human beings on the field under emergencies, where there might be several dangerous areas that threaten the safety of human beings, People need to be guided out of the field while keeping away from those dangerous areas. Fig. 1.1 depicts such an example scenario. We characterize the navigation problem as a path planning problem and present its assumptions, objectives as follows.

Assumptions: We assume an emergent field containing several areas of dangers, as the red areas shown in Fig. 1.1 The dangerous areas might emerge, disappear, expand, or shrink as the time passes. The number of dangerous areas at any time is finite.

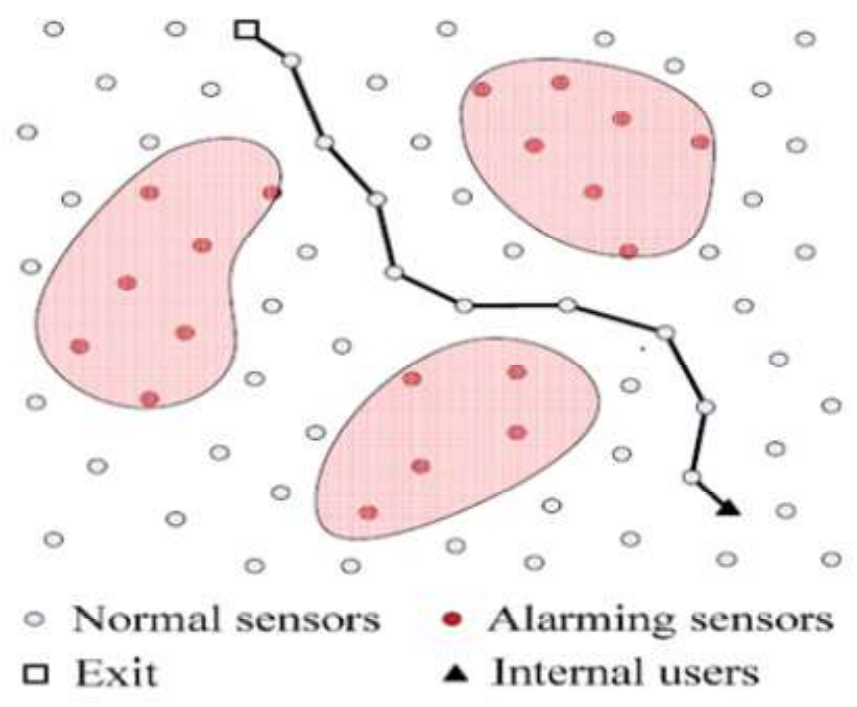

Figure : Navigation for trapped user

A sensor network system is deployed in the field, where each sensor is able to detect the dangers distributed over the field. The sensor node triggers a "yes" alarm if it resides in the dangerous area(highlighted ovals) and triggers "no" if outside dangerous area. Thus, the boundary of a dangerous area can be outlined by pairs of neighboring temperature sensors with different outcomes.

Each user carries a communicating device that can talk with system. By measuring the strength and direction of wireless signals, the user is able to track any targeted sensor node. Thus, the navigating route can be interpreted as a sequence of nodes.

Objectives: The objective of a successful navigation is to plan a path for each user to one or more pre known exits on the field that lead to safe departure, bypassing all the dangerous areas. In Fig. 1.1 there is an exit in the fields that users are required to lead to. We depict such an example route that leads the internal user to the exit. The navigation process is carried out in a fully distributed manner without any dedicated central agents like sinks. Each user is handoff guided by sensors along the entire route.

Problem Definition: To provide optimal safety for every smart phone user in any emergency situation by providing optimal path towards exits, System takes the hazard levels of emergencies and the evacuation capabilities of exits into account and provides the mobile users the safest navigation paths accordingly. 


\section{Present Work:}

As emergency or dangerous situations change, it becomes necessary to frequently update the route plans for the guided users. Path dynamics in traditional packet routing process typically occurs between delivering different packets, while such dynamics in the navigation process might exist all along for guiding single individuals [3]. People may even move backward to seek broader opportunities under varied situations.

There have been attempts made at guiding navigation using WSNs.

Most, if not all, existing approaches assume the availability of locations on each sensor node[1]. Knowing the locations of dangerous areas, the sensor network can perform easy and efficient route calculations to navigate internal users out of the emergency area[1]. The location information, however, may not always be available in many realistic situations where emergency guidance are needed, for example, in an underground tunnel or coal mine, a complex indoor area, and so on. The requirement of location information largely constrains the applicability of existing approaches to locationfree environments[5]. In addition, existing approaches usually do not specifically consider the impact of variations of dangerous areas, for example, the expansion, shrinking, or disappearing of areas which is deemed dangerous[5]. In reality, such variations often degrade the effectiveness of existing designs or even overwhelm them.

So far they haven't take evacuation capability of exits into account which makes big impact in real time.

\section{Proposed Work:}

We have arrive at the plain fact that a practical and efficient emergency navigation scheme should be situation-aware, which means that we should take into consideration both the hazard levels of concurrent emergencies and the evacuation capabilities of exits.

Despite its importance, on the down side, we capitalize that it is not straightforward to design such a situation aware emergency navigation. It is nontrivial to directly extending existing methods which inherently aim at navigating users along the paths with equal distances to emergencies. The main challenge here is how to define the safety properly, incorporating the impacts of both different hazard levels of emergencies and different capabilities of the exits at the same time.

\section{Technology used in the process:}

System takes the hazard levels of emergencies and the evacuation capabilities of exits into account and provides the mobile users the safest navigation paths accordingly. Here we use WSN as system's backbone, along with Arduino-Uno chipset, Temperature sensors and $\mathrm{Wi}-\mathrm{Fi}$ for user-arduino Communication and smartphones.

\section{- Arduino IDE}

The Arduino Integrated Development Environment (IDE) is a cross-platform application (for Windows, macOS, Linux) that is written in the programming language Java. It originated from the IDE for the languages Processing and Wiring. It includes a code editor with features such as text cutting and pasting, searching and replacing text, automatic indenting, brace matching, and syntax highlighting, and provides simple one-click mechanisms to compile and upload programs to an Arduino board. It also contains a message area, a text console, a toolbar with buttons for common functions and a hierarchy of operation menus. The source code for the IDE is released under the GNU General Public License, version 2. The Arduino IDE supports the languages $\mathrm{C}$ and $\mathrm{C}++$ using special rules of code structuring.

\section{Web technology:}

Hypertext Markup Language (HTML) is the standard markup language for creating web pages and web applications. With Cascading Style Sheets (CSS) and JavaScript, it forms a triad of cornerstone technologies for the World Wide Web.Web browsers receive HTML documents from a web server or from local storage and render the documents into multimedia web pages.

HTML describes the structure of a web page semantically and originally included cues for the appearance of the document. HTML elements are the building blocks of HTML pages. With HTML constructs, images and other objects such as interactive forms may be embedded into the rendered page.

\section{Arduino:}

The arduino is a series of small single-board computers developed in the United Kingdom by the 
arduino Foundation to promote the teaching of basic computer science in schools and in developing countries. The original model became far more popular than anticipated,selling outside of its target market for uses such as robotics. Peripherals (including keyboards, mice and cases) are not included with the arduino.

\section{Setup:}

We have Installed the Arduino IDE, it supports varying platform, windows, Linux and Mac. We have used Windows platform. Once Arduino is installed it is time to test ESP8266 wifi Shield. We have connected the Arduino board using USB cable to your computer for continuous power suply. The computer should detect this automatically.

- Select the correct board from : Tools->Board-> Generic ESP8266 Module.

- The next step is to select the port where your Arduino board is connected to, so go to : Tools->Port.

- Once you have this components connected, you can test the connection and the board by loading the program sketch :

Select File->Examples->basics ->blink.

This will open the main sketch, you can click on the "Upload" button to upload your sketch. If all goes well then your module will work perfectly.

The temperature sensor LM35 have 3 legs, the first leg is VCC, you can connect this to the $3.3 \mathrm{~V}$ (ESP8266 board's output is $3.3 \mathrm{~V}$ ). The middle leg is Vout (where the temperature is read from, you can connect this to the analog input of the

ESP8266 pin AD0, this is located at the top right hand side of the board as shown in picture. And the right leg should be connected to the ground. Now your circuit is complete.

\section{CONCLUSION:}

This system conducts the first work on situationaware emergency navigation by considering a more general and practical problem, where emergencies of different hazard levels and exits with different evacuation capabilities may coexist. We first model the situation-aware emergency navigation problem and formally define the safety of a navigation path.
We are currently devoting to conducting a small-scale system prototype under more complex scenarios. In the future, we would like to explore modeling the hazard speed in the context of emergency navigation. We also plan to cooperate with the local Fire Department to test our prototype, e.g., in the firefighting exercises, to provide more evidences on the real effects on user safety in real scenarios.

\section{ACKNOWLEDGEMENT:}

We are the authors from the University of Pune wish to acknowledge the contribution of all support staff of Pune Vidyarthi Griha's College of Enginering (Computer Department) who have helped us for the success of this research.

\section{REFERENCES}

1) Amundson and X. D. Koutsoukos, "A survey on localization for mobile wireless sensor networks," in Mobile Entity Localization and Tracking in GPS-less Environnments. Springer, 2009, pp. 235-254.

2) T.He, P. Vicaire, T. Yan, L. Luo, L. Gu, G. Zhou, R. Stoleru, Q. Cao, J. A. Stankovic, and T. Abdelzaher, "Achieving real-time target tracking using wireless sensor networks," in Real-Time and Embedded Technology and Applications Symposium, 2006. Proceedings of the $12^{\text {th }}$ IEEE. IEEE, 2006, pp. 37-48.

3) - A. Brown, J. Griesbach, B. Bockius, and T. Boult, "Gps tracking location-based service using wristwatch geozigbee sensors," in Proceedings of the ION National Technical Meeting, 2007.

4) D. Capriglione, L. Ferrigno, E. D'Orazio, V. Paciello, and A. Pietrosanto, "Reliability analysis of rssi for localization in small scale wsns," in Instrumentation and Measurement Technology Conference (I2MTC), 2012 IEEE International. IEEE, 2012, pp. 935-940.

5) S. Ray, J. Kumar, S. Sen, and J. Nath, "Modified distance vector routing scheme for a manet," in The National Conference on Communications, Kanpur, India, 2007, pp. 197-202.

6) D. Niculescu and B. Nath, "Dv based positioning in ad hoc networks," Telecommunication Systems, vol. 22, no. 1-4, pp. 267-280, 2003. 
7) M. L. Sichitiu and V. Ramadurai, "Localization of wireless sensor networks with a mobile beacon," in Mobile Ad-hoc and Sensor Systems, 2004 IEEE International Conference on. IEEE, 2004, pp. 174-183.

8) R. Priwgharm and P. Chemtanomwong, "A comparative study on indoor localization based on rssi measurement in wireless sensor network," in Computer Science and Software Engineering (JCSSE), 2011 Eighth International Joint Conference on. IEEE, 2011, pp. 1-6.

9) A. H. Sayed, A. Tarighat, and N. Khajehnouri, "Network-based wireless location: challenges faced in developing techniques for accurate wireless location information," Signal Processing Magazine, IEEE, vol. 22, no. 4, pp. 24-40, 2005.

10) G. Sun, J. Chen, W. Guo, and K. R. Liu, "Signal processing techniques in network-aided positioning: a survey of state-of-the-art positioning designs," Signal Processing Magazine, IEEE, vol. 22, no. 4, pp. 12-23, 2005.

11) S. Kim, S. Kim, and D.-S. Eom, "Rssi/lqi-based transmission power control for body area networks in healthcare environment," Biomedical and Health Informatics, IEEE Journal of, vol. 17, no. 3, pp. 561-571, 2013.

12) N. Baccour, A. Koubaa, L. Mottola, M./A. Z'ũniga, H. Youssef, C. A. Boano, and M. Alves, "Radio link quality estimation in wireless sensor networks: A survey," ACM Trans. Sen. Netw., vol. 8, no. 4, pp. 34:1-34:33, Sep. 2012. [Online]. Available:

http://doi.acm.org/10.1145/2240116.2240123

13) M. Rondinone, J. Ansari, J. Riihijarvi, and P. M"ah"onen, "Designing a reliable and stable link quality metric for wireless sensor networks," in Proceedings of the workshop on Real-world wireless sensor networks, ser. REALWSN '08. New York, NY, USA: ACM, 2008, pp. 6-10. 